\title{
Inguinal Lymph Node Metastasis of a Primary Serous Papillary Carcinoma of the Peritoneum One Year after CRS and HIPEC
}

\author{
Shadi Katou Mathilde Feist Wieland Raue Johann Pratschke Beate Rau Andreas Brandl \\ Department of Surgery, Campus Virchow-Klinikum and Charité Campus Mitte, Charité - Universitätsmedizin Berlin, Berlin, Germany
}

\section{Keywords}

Primary peritoneal serous papillary carcinoma .

Inguinal lymph node metastasis - Cytoreductive surgery . Hyperthermic intraperitoneal chemotherapy

\section{Summary}

Background: Primary peritoneal serous papillary carcinoma is a rare malignant epithelial tumor which was first described in 1959. Peritoneal serous papillary carcinoma arises from the peritoneal epithelium and originates from a single or multicentric focus of the peritoneum involving the peritoneum of the abdomen and pelvis. The involvement of retroperitoneal lymph nodes occurs in $64 \%$ of the patients diagnosed with this malignancy. So far, there is no report about inguinal lymph node metastasis in this disease. Case Report: We present a rare case of a 63-year-old female patient who developed singular inguinal lymph node metastasis 1 year after cytoreductive surgery and hyperthermic intraperitoneal chemotherapy due to peritoneal serous papillary carcinoma. The lymph node metastasis was found by computed tomography (CT) scan and was resected and histologically confirmed. The postoperative course was uneventful, and the patient was discharged on postoperative day 1 . The last CT scan 24 months after initial cytoreduction and 12 months after lymph node resection showed no further tumor recurrence. Conclusion: This case report should raise the awareness of potentially unexpected presentation of extraperitoneal metastasis and highlights the importance of patient follow-up including clinical examination and CT scans of thorax/abdomen/ pelvis following a systematic schedule.

(c) 2018 S. Karger GmbH, Freiburg

\section{KARGER}

() 2018 S. Karger GmbH, Freiburg

\section{Introduction}

Primary peritoneal serous papillary carcinoma (PPSPC) is an uncommon malignancy emerging in the abdominal or pelvic peritoneal lining and causing severe ascites. It was first described by Swerdlow [1] in 1959 and presents predominantly in elderly and post-menopausal women. It is a rare disease with an incidence of 4.62 per million inhabitants in the USA, as presented in a large examination by Goodman and Shvetsov [2]. The pathological fact that it was nearly indistinguishable from papillary serous carcinoma of the ovary made it difficult to differentiate and diagnose. Therefore, a set of specific diagnostic criteria was established by the Gynecologic Oncology Group in 1993, although by that time less than 250 cases of PPSPC were reported [3]. The consensus meeting agreed on the criteria illustrated in table 1.

The establishment of these criteria led to a notable increase in the diagnosis of PPSPC in the past years and to a better understanding of its presentation and treatment. Nevertheless, much remains indefinite about this malignancy.

In recent years, a remarkable increase of the diagnostic frequency has been noted due to the better understanding of the clinical presentation und behavior of PPSPC [4]. Because of the biological and pathological similarities that PPSPC shares with ovarian serous tumors, it is likely that both malignancies have a common molecular pathogenesis arising from mesothelial cells under Mullerian influence [2]. However, the pathogenesis and etiology of this disease still remains largely unknown.

The recommended therapy includes a doublet intravenous chemotherapy with taxol and platin, which has shown greater response rates in several studies than previous chemotherapy protocols with alkylating agents. A retrospective multicenter study performed by Unal et al. [5] including patients with PPSPC showed a superior median overall survival for patients treated with the combination cisplatin/paclitaxel compared to carboplatin/paclitaxel (70.3 vs. 41 months) as first-line treatment. During the last decades 
and after the development of cytoreductive surgery (CRS), more patients were treated with a combination of CRS and hyperthermic intraperitoneal chemotherapy (HIPEC) in addition to intravenous chemotherapy, thus showing remarkable results. Nam et al. [6] showed a significantly longer median survival in patients who had optimal cytoreduction (largest residual tumor mass $<1.0 \mathrm{~cm}$ ) than those who had suboptimal cytoreduction (41 vs. 10 months, respectively).

Despite the peritoneal spreading of the tumor, it is known that pelvic and para-aortic lymph nodes can also be affected by PPSPC with a frequency of up to $72 \%$ [7]. In case of lymph node involvement, Dubernard et al. [8] showed that lymphadenectomy seemed to be beneficial in a small series of 19 patients and reported an overall survival of $83 \%$ after 3 years. In few cases breast and/or axillar lymph node metastases have been reported; however, it is of great importance to differentiate these from primary tumors of the breast $[9,10]$.

\section{Case Report}

We report on a 63-year-old female patient with PPSPC who had no further medical history, previous surgeries, or comorbidities. The patient presented to the emergency room of a nearby local hospital with abdominal distension lasting for 3 weeks as well as pain in the lower abdomen, especially postprandial and while standing. After diagnostic laparoscopy and histopathologic diagnosis of PPSPC the tumor was declared inoperable and palliative chemotherapy was recommended. The patient was referred to our department for a second opinion. We repeated diagnostic laparoscopy to verify the option for CRS and HIPEC and staged a Peritoneal Cancer Index (PCI) of 12 with little involvement of the small bowel mesentery (fig. 1). The recommendation of the interdisciplinary tumor board was in favor of CRS and HIPEC. Primary resection included infragastric omentectomy, appendectomy, pelvic peritonectomy, peritonectomy of the abdominal wall, and mesentery of the small bowel in January 2015. The duration of the procedure was $243 \mathrm{~min}$, and complete cytoreduction could be achieved (CC 0/1). There were no signs of iliac lymph node metastases or ovarian metastases. HIPEC was conducted through a closed circulation system with $75 \mathrm{mg} / \mathrm{m}^{2}$ cisplatin and $15 \mathrm{mg} / \mathrm{m}^{2}$ doxorubicin over $60 \mathrm{~min}$. The cancer antigen 125 (CA-125) dropped from 22 to $8.4 \mathrm{kU} / \mathrm{l}$ after CRS and HIPEC as a sign of cytoreduction. The diagnosis of PPSPC was histologically confirmed and showed an MIB-1 of 25\%. The postoperative course was uneventful. The patient was discharged 8 days after surgery and was enrolled as an out-patient in our clinic for follow-up. After surgical treatment the case was discussed in our interdisciplinary tumor board. The recommendation was to surveil the patient with clinical examinations every 3 months and computed tomography (CT) scan every 6 months, with no further chemotherapy.

CT scan 6 months after CRS and HIPEC excluded tumor recurrence, whilst CT scan after 12 months presented a suspicious inguinal lymph node without peritoneal tumor recurrence (fig. 2). Clinically, the patient reported neither signs of abdominal discomfort nor palpable solid mass nor ascites. The CA-125 slightly increased to $9.1 \mathrm{kU} / \mathrm{l}$. The case was discussed for another time at the interdisciplinary tumor conference, which recommended the resection of the inguinal lymph node sized $25 \times 15 \times 10 \mathrm{~mm}$. This resection was carried out in February 2016. The histological findings confirmed the origin of PPSPC. The postoperative CA-125 was $7.5 \mathrm{kU} / 1$ in May 2016. The clinical course of the patient was uneventful, presenting without further tumor recurrence 2 years after CRS and HIPEC in January 2017. The interdisciplinary tumor conference recommended again in favor of a watch-and-wait strategy without indication for systemic chemotherapy. The patient is currently still alive and well; her last follow-up was in January 2017, i.e. 2 years after CRS and HIPEC. Her weight is constant, physical examination was unremarkable, and CT scan showed no changes to the previous follow-up and no tumor recurrence.
Table 1. Criteria for primary peritoneal serous papillary carcinoma

1 Both of the ovaries are either absent, normal in size, or enlarged by a benign process

2 The bulk of the tumor is on the peritoneum, and extraovarian involvement should be greater than on the ovarian surface

3 In case of an ovarian involvement, this should be either confined to the surface epithelium, with absence of cortical invasion or involving the ovarian surface and the underlying cortical stroma, but less than $5 \times 5 \mathrm{~mm}$ in diameter

4 Histological and cytological characteristics of the tumor are predominantly of the serous type

\section{Discussion}

PPSPC is a rare malignant epithelial tumor found predominantly in elderly and post-menopausal women.

The most characteristic presentation of PPSPC is diffuse involvement of the peritoneum with massive ascites, mesenteric and omental nodules, or even omental cake. It rarely metastasizes to the liver, lung, or brain [11]. The median survival time from the diagnosis of PPSPC has been reported to be around 11-17 months [12]. To our knowledge, we are describing the first case of inguinal lymph node metastasis of PPSPC.

So far, due to its rarity, PPSPC has been mostly discussed und studied in the literature by means of single case reports. It has shown a variety of clinical presentation and spreading patterns. Ranging from a single colonic mass [13] to distant lymph nodes without intraperitoneal dissemination [14], the understanding of the clinicopathological behavior of PPSPC has been much of a challenge.

The involvement of lymph nodes and the spreading pattern of the malignant cells of PPSPC through the lymphatic vessel and lymph nodes have been poorly defined. Steinhagen and Sehouli [15] noted in their review of existing literature that around twothirds of patients suffering from PPSPC appear to have lymphatic metastasis of the retroperitoneum. In a further three cases a metastatic spreading to the breast and/or axillar lymph nodes has been described. In these cases, it was important to distinguish PPSPC from primary breast cancer and its metastases [10]. Our case did not show any retroperitoneal lymph node metastases, neither at the initial CRS and HIPEC procedure nor in the follow-up CT scans.

The lymph drainage pattern of the peritoneum is complex and differentiates between parietal and visceral peritoneum. Whereas the parietal part drains to parietal lymph nodes lying behind the peritoneum and leading to axillary, superficial inguinal and lumbar lymph nodes, lymph of the visceral peritoneum joins preaortic groups of nodes surrounding the abdominal aorta and drains via the cisterna chyli to the thoracic duct [16].

Inguinal lymph nodes are a common site of metastasis for malignancies located in the pelvis, lower extremities, and back. The deep inguinal lymph nodes receive afferents from superficial inguinal nodes, whereas those receive lymph from the lower extremities, external genitalia, anus, and infraumbilical region of the anterior abdominal wall. Tumors that frequently metastasize to inguinal lymph nodes are malignant lymphoma, squamous cell carcinoma 
Fig. 1. Intraoperative picture demonstrating primary peritoneal serous papillary carcinoma with ascites.

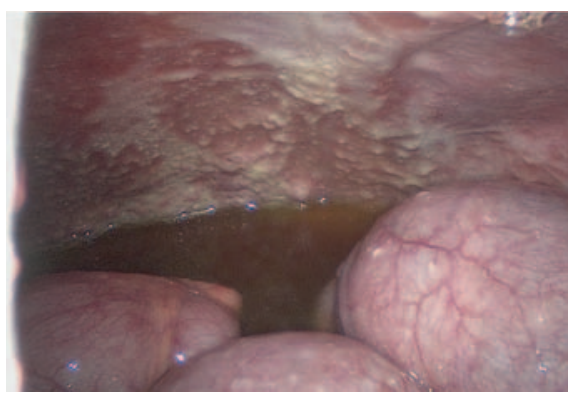

Fig. 2. Computed tomography image at 1-year follow up; the right inguinal lymph node is presented enlarged.

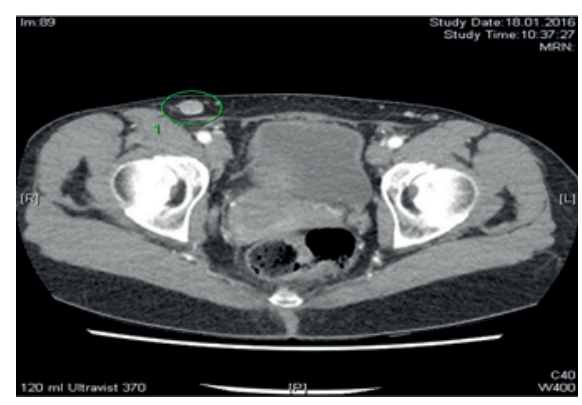

of the vulva or penis, and malignant melanoma of the legs. The most common gastrointestinal tumors to metastasize to inguinal nodes are squamous cell carcinoma of the anus and adenocarcinoma of the lower third of the rectum [17]. None of these tumors were present in our patient.

In our case, we reported on a 63-year-old patient with PPSPC who underwent CRS and HIPEC and had a good clinical course. CRS and HIPEC was carried out in January 2015, with an initial PCI of 12. 1 year later, the patient developed an inguinal lymph node metastasis without any intra-abdominal tumor recurrence. The lymph node was surgically resected in February 2016, and histology confirmed the origin of PPSPC. A watch-and-wait strategy was decided without indication for systematic chemotherapy; 2 years after CRS and HIPEC the patient is alive and well, showing no further recurrence of tumor.

We consider this finding as important since it is the first case of inguinal lymph node metastasis of PPSPC ever reported. It highlights the importance of follow-up examinations of patients with PPSPC. Despite the absence of intra-abdominal tumor recurrence, our patient developed inguinal lymph node metastasis. We recommend postoperative routine examinations with imaging of the thorax, abdomen, and pelvis (CT scan or magnetic resonance imaging
(MRI)) every 6 months. Postoperative routine examinations help to detect tumor recurrence early and in a resectable stage. This report demonstrates that tumor recurrence can also appear in rather unlikely localizations of lymphatic drainage such as the inguinal lymph nodes and that follow-up examinations should routinely include physical examinations of these regions.

\section{Conclusion}

In conclusion, PPSPC is a rare malignancy with only little understanding of its origin and behavior; therefore, its pattern is not fully known yet. The aim of this case report is to raise awareness of a potentially unexpected presentation of extraperitoneal metastasis and highlights the importance of patient follow-up including clinical examination and imaging of thorax/abdomen/pelvis (CT scan or MRI) following a systematic schedule.

\section{Disclosure Statement}

The authors have neither financial relationships nor grants nor sponsoring of equipment or drugs to disclose. No conflict of interest.

\section{References}

1 Swerdlow M: Mesothelioma of the pelvic peritoneum resembling papillary cystadenocarcinoma of the ovary case report. Am J Obstet Gynecol 1959;77:197-200.

2 Goodman MT, Shvetsov YB: Rapidly increasing incidence of papillary serous carcinoma of the peritoneum in the United States: fact or artifact? Int J Cancer 2009; 124:2231-2235.

3 Bloss JD, Brady MF, Liao SY, Rocereto T, Partridge EE, Clarke-Pearson DL; Gynecologic Oncology Group Study: Extraovarian peritoneal serous papillary carcinoma: a phase II trial of cisplatin and cyclophosphamide with comparison to a cohort with papillary serous ovarian carcinoma - a Gynecologic Oncology Group Study. Gynecol Oncol 2003;89:148-154.

4 Halperin R, Zehavi S, Langer R, Hadas E, Bukovsky I, Schneider D: Primary peritoneal serous papillary carcinoma: a new epidemiologic trend? A matched-case comparison with ovarian serous papillary cancer. Int J Gynecol Cancer 2001;11:403-408.

5 Unal OU, Oztop I, Yazici O, et al: Treatment and prognostic factors in primary peritoneal carcinoma: a multicenter study of the Anatolian Society of Medical Oncology (ASMO). Oncol Res Treat 2014;37:332-338.
$6 \mathrm{Nam}$ JH, Kim YM, Jung MH, et al: Primary peritoneal carcinoma: experience with cytoreductive surgery and combination chemotherapy. Int J Gynecol Cancer 2006; $16: 23-28$.

7 Eltabbakh GH, Mount SL: Lymphatic spread among women with primary peritoneal carcinoma. J Surg Oncol 2002;81:126-131.

$>$ Dubernard G, Morice P, Rey A, et al: Lymph node spread in stage III or IV primary peritoneal serous papillary carcinoma. Gynecol Oncol 2005;97:136-141.

$\checkmark 9$ Recine MA, Deavers MT, Middleton LP, Silva EG, Malpica A: Serous carcinoma of the ovary and peritoneum with metastases to the breast and axillary lymph nodes - a potential pitfall. Am J Surg Pathol 2004;28: 1646-1651.

10 Sun JY, Gebre W, Dong YM, Shaun X, Robbins R, Podrumar A: Primary peritoneal carcinoma metastasizing to breast: a single case report and literature review from clinic to biology. Cancer Biol Med 2016;13: 389-395.

11 Sakakibara Y, Endo S, Yoshida Y, Tanaka Y, Hashimoto T, Ohara T: A case of serous surface papillary carcinoma of the peritoneum metastatic to the brain (Article in Japanese). No Shinkei Geka 2011;39:607610.
12 Roh SY, Hong SH, Ko YH, et al: Clinical characteristics of primary peritoneal carcinoma. Cancer Res Treat 2007;39:65-68.

13 Kim HS, Sung JY, Park WS, Kim YW: Primary peritoneal serous papillary carcinoma presenting as a single colonic mass without peritoneal dissemination. Intern Med 2013;52:227-232.

14 Yonehara T, Yamaguchi T, Azuma M, Minobe S, Sakuragi N: A case of primary serous papillary carcinoma with unusual clinical presentation: distant lympho nodes metastasis without peritoneal dissemination. Arch Gynecol Obstet 2008;278:579-583.

15 Steinhagen PR, Sehouli J: The involvement of retroperitoneal lymph nodes in primary serous-papillary peritoneal carcinoma. A systematic review of the literature. Anticancer Res 2011;31:1387-1394.

16 Parungo CP, Soybel DI, Colson YL, et al: Lymphatic drainage of the peritoneal space: a pattern dependent on bowel lymphatics. Ann Surg Oncol 2007;14:286298.

17 Hajdarbegovic E, Balak D: Images in clinical medicine. Metastatic inguinal lymphadenopathy. N Engl J Med 2012;366:1526. 